\title{
Bimodal Morphological Analyses of Native and Engineered Tissues
}

Sasan Jalili-Firoozinezhad ${ }^{\#}$, Ivan Martin*, Arnaud Scherberich

Departments of Biomedicine, of Biomedical Engineering and of Surgery, University Hospital Basel, University of Basel, Hebelstrasse 20, CH-4031 Basel, Switzerland

${ }^{\#}$ Current address: Wyss Institute for Biologically Inspired Engineering at Harvard University, 3 Blackfan Circle, Boston MA 02115, USA. sasan.jalili@wyss.harvard.edu, Tel: +1 617432 7019

\section{*Corresponding Author:}

Ivan Martin, Ivan.martin@usb.ch

Department of Biomedicine, University Hospital Basel, University of Basel, Hebelstrasse 20, CH-4031 Basel, Switzerland

Tel: +41612652384

Fax: +41612652350

Keywords: histology; scanning electron microscopy; extracellular matrix; morphology; tissue structure 


\begin{abstract}
Assessing the morphological features of native and engineered tissues is pivotal to evaluate their degree of development and to identify possible structure-function relationships. Conventional histological or immunohistochemical imaging of stained sections provides limited information about their architecture. Scanning electron microscopy (SEM) yields submicrometric resolution images of tissues, but typically cannot be associated with the morphological structures identified by histology. The aim of this study was to establish a technique based on SEM analysis of sections of paraffin-embedded tissues prepared for histological processing (Histo-SEM) for the assessment of morpho-architectural properties of native and engineered tissues. Histo-SEM was performed on sections of cartilaginous, bone and fibrous tissues, native or engineered, in parallel with histological/immunohistochemical staining. Histo-SEM technique allowed evaluating morpho-architectural features typically unreachable by conventional histological staining, like (i) the extent of cartilage maturation based on collagen fibers' diameter and orientation, (ii) the formation of bone tissue/osteoid based on the presence of nanoscale-dense matrix structures, and (iii) tissue integration and vascularization based on collagen fibers' density and average vessel walls thickness of fibrous tissue growing within ectopically implanted porous materials. In conclusion, Histo-SEM allows integrating bimodal morphological assessments of native or engineered tissues and deriving complementary qualitative and quantitative parameters related to their structural organization and level of maturation.
\end{abstract}




\section{Introduction}

Histology is an indispensable technique in biology and medicine to investigate the microscopic anatomy of tissues. It is routinely performed and enhanced by the use of histological stains (histochemistry) or immunostaining tools (immunohistochemistry), and allows for the microscopic visualization and identification of tissue structures as various as nucleic acids, matrix or cellular proteins, lipids of the cellular membranes. A limitation of the histological evaluations, however, is an unsatisfactory spatial resolution of imaging in order to provide sub-micrometric pictures and quantitative data measuring features of tissue maturation, organization or vascularization [1-4]. Previous efforts to monitor submicrometric differences between ECM from constructs with different chondrogenic capacity, as well as different extent of host interaction were limited when using histological processing only $[5,6]$.

Other imaging strategies are available to monitor the structural organization and quality of native and engineered tissues. For instance, X-ray microcomputed tomography $(\mu \mathrm{CT})$ has been identified as a nondestructive tool to evaluate 3D structure of engineered tissues, especially in bone mineralization studies. However, this technique is limited to radio-opaque materials (e.g. calcified bone) and thus provides very limited information about radiolucent materials (e.g. polymers and soft ECMs) [2]. While other techniques such as spectral ultrasound imaging are applicable to soft tissue, some features of cells and tissue smaller than micrometric resolution are missed [3]. Two-photon and multiphoton microscopy, as well as visualizing the second harmonic generation signals, can potentially provide information at sub-micrometric level and detect ECM fibers in tissue, but these techniques suffer from light scattering and are not recommended for complex structures [4,7,8]. Among available strategies, scanning electron microscopy (SEM), allows for high resolution analysis of various features, from the visualization of the nano-sized architecture of extracellular matrix (ECM) 
fibers to the morphology of cells [9-11]. However, this imaging modality is only applicable to the surface of native and engineered tissues, unless the sample is snap frozen or lyophilized and manually fractioned to reveal the inner part of the sample $[12,13]$. Such harsh fractionation techniques often lead to major deterioration of the structures at the exposed surface of the sample. Moreover, SEM is a destructive method which requires a specific processing, making the analyzed samples unavailable for further analysis by other techniques. The possibility to precisely define the correspondence between the observed structures and the specific types of tissues and cells, typically identified by histological or immunohistochemical staining, would reinforce the use of SEM to assess morphological features of engineered and native tissue samples [14,15].

The aim of this study was therefore the development and validation of an approach, based on the use of SEM on histological sections of the tissue to be analyzed, here referred to as histology-linked-SEM (Histo-SEM) technique. Histological sections of paraffin-embedded tissues were analyzed by light microscopy (LM) and SEM in parallel. The validation was performed on samples from three different tissue types: (a) cartilage tissue engineered in vitro by macromass pellet cultures, with native cartilage used as control, (b) bone tissue engineered in vivo by stromal vascular fraction cells seeded in ceramic scaffolds, and (c) fibrous tissue grown within the pores of in vivo implanted cell-free macroporous sponges. Using these three tissues as exemplifying contexts of implementation, we illustrate how Histo-SEM allowed integrating qualitative and quantitative data of different nature to characterize tissue structure and morphology.

\section{Materials and Methods}

\subsection{Sample preparation}


Engineered cartilage constructs. Cartilage tissue samples were obtained from a recent study [6]. To fabricate engineered cartilage constructs, clonal strains of freshly isolated human articular chondrocytes with different chondrogenic capacities were suspended in chondrogenic medium $\left(10 \mu \mathrm{g} \cdot \mathrm{ml}^{-1}\right.$ insulin, $5.5 \mu \mathrm{g} \cdot \mathrm{ml}^{-1}$ transferrin, $5 \mathrm{ng} \cdot \mathrm{ml}^{-1}$ selenium, 0.5 mg.ml ${ }^{-1}$ human serum albumin, $0.1 \mathrm{mM}$ ascorbic acid 2-phosphate, $10^{-7} \mathrm{M}$ dexamethasone, $4.7 \mu$ g.ml linoleic acid and 10 ng. $\mathrm{ml}^{-1}$ TGF $\beta$ ), diluted at $10^{6}$ cell. $\mathrm{ml}^{-1}$, distributed in $1.5 \mathrm{ml}$ conical tubes ad centrifuged at $1100 \mathrm{rpm}$ for $3 \mathrm{~min}$ to form 3D pellets. Pellets were then cultured for 14 days to generate tissues consisting of fibrotic or cartilaginous ECM. As reference, human articular cartilage from the femoral lateral condyles was processed as previously described [16].

Engineered bone constructs. Bone tissue samples were obtained from previously described osteogenic constructs [17]. To generate engineered bone constructs, stromal vascular fraction cells $\left(8.6 \times 10^{5}\right.$ cells.ml $\left.{ }^{-1}\right)$ from human adipose tissue were perfused at the velocity of $3 \mathrm{ml} . \mathrm{min}^{-}$ ${ }^{1}$ through porous hydroxyapatite ceramic scaffolds (ENGIpore; Fin-Ceramica Faenza, Faenza, Italy, http://www.finceramica.it) with average porosity of $83 \%$ and in shape of $8 \mathrm{~mm}$ diameter x $4 \mathrm{~mm}$ thickness disks for 5 days by using a 3D perfusion culture system (Cellec Biotek AG, Basel, Switzerland, http://www.cellecbiotek.com). Constructs were perfused with $\alpha$-MEM medium containing $10 \%$ fetal bovine serum (FBS) supplemented with 5 ng.ml ${ }^{-1}$ fibroblast growth factor-2 (FGF-2) during the seeding and afterwards. Constructs were subsequently implanted ectopically in nude mice (CD-1 nu/nu, 1-month old; Charles River Laboratories, Wilmington, MA, http://www.criver.com) for 8 weeks. Then, the samples were harvested, fixed in $4 \%$ formalin, decalcified by incubation with Osteodec (Bio-Optica) and histologically processed for further investigations.

Implanted cell-free sponges. Samples with different levels of in vivo engraftment and vascular ingrowth were selected from a recent study [5]. In short, egg white and collagen type I 
macroporous sponges were chemically crosslinked with $\mathrm{N}^{\prime}$-ethylcarbodiimide hydrochloride (Sigma-Aldrich) and subcutaneously implanted in nude mice (CD-1 nu/nu, 4-6 weeks old, Charles-River, Sulzfeld, Germany). 30 days after implantation, scaffolds were explanted and kept for further analyses.

\subsection{Sample analyses}

Histological analysis. Cartilage, bone constructs and explanted sponges were fixed overnight in $4 \%$ formalin. For bone samples, an additional step of decalcification was introduced, by incubation for $3 \mathrm{~h}$ with Osteodec (Bio-Optica, Milan, Italy, http://www.bio-optica.it) at $37^{\circ} \mathrm{C}$ on an orbital shaker. Specimens were subsequently washed with double distilled water and dehydrated with increasing ethanol concentration in solution in a tissue processor machine. Paraffin blocks were then generated and $7 \mu \mathrm{m}$ sections were performed. Sections were stained with hematoxylin and eosin (H\&E, Sigma), with Safranin-O (Sigma) for sulfated glycosaminoglycans, or with Masson's Trichrome (Kit Trichrome de Masson-Vert lumière, Réactifs RAL, www.ral-diagnostics.fr). To visualize blood vessels, sections were processed by immunohistochemistry with antibodies to CD31 (Abcam, ab32457, 1:200 (V/V)), Vectastain ABC kit, followed by Fast Red kit (both from Dako) and counterstained with hematoxylin. LM analyses used an optical microscope (Olympus, BX63 with Olympus DP72 digital camera).

Histology-Scanning Electron Microscopy analysis (Histo-SEM). Following paraffin embedding and sectioning, tissue slides were dried in a $37^{\circ} \mathrm{C}$ oven for $18 \mathrm{~h}$, deparaffinized by two rinsing steps of $15 \mathrm{~min}$ in Xylene (Sigma), rinsed in absolute ethanol (Merck) for $30 \mathrm{~min}$, dried in a Critical Point Dryer (CPD, Baltec 030, Leica, Germany), sputter-coated with gold and observed with a scanning electron microscope (FESEM, FEI Nova NanoSEM 230, USA) under an operating voltage of $15 \mathrm{kV}$. Average fiber-to-fiber length, fiber diameter and 
thickness of vessel's lumens were determined from at least 100 measurements on SEM micrographs with Image Analyzer software (Image J 1.44p). Image processing method using Image $\mathbf{J}$ software was employed to analyze the distribution of fiber orientations, ranging from $-90^{\circ}$ to $90^{\circ}$, where $0^{\circ}$ was defined as the vertical direction of each image.

Alternatively, Histo-SEM analysis was carried out on sections previously processed for histological staining and imaged by LM. In those cases, however, since the sections needed to be sputter-coated with gold, further analysis by LM was not recommended.

\subsection{Statistical analysis}

Data were expressed as mean \pm standard deviation and statistical analysis was accomplished by one-way analysis of variance and independent Student's t-test using SPSS 16.0 software. Post-hoc analyses were performed with Tukey's honest significant difference (HSD) tests. P values of less than 0.05 were considered to be statistically significant.

\section{Results and Discussion}

Unless otherwise stated, the structure and morphology of native and bioengineered samples were inspected qualitatively and quantitatively by optical and electron microscopes on 2 serial sections (Figure 1). For samples in which SEM analyses were performed on stained sections, a single tissue section went first through histological processing and LM and was thereafter used for CPD and SEM imaging as described above. 


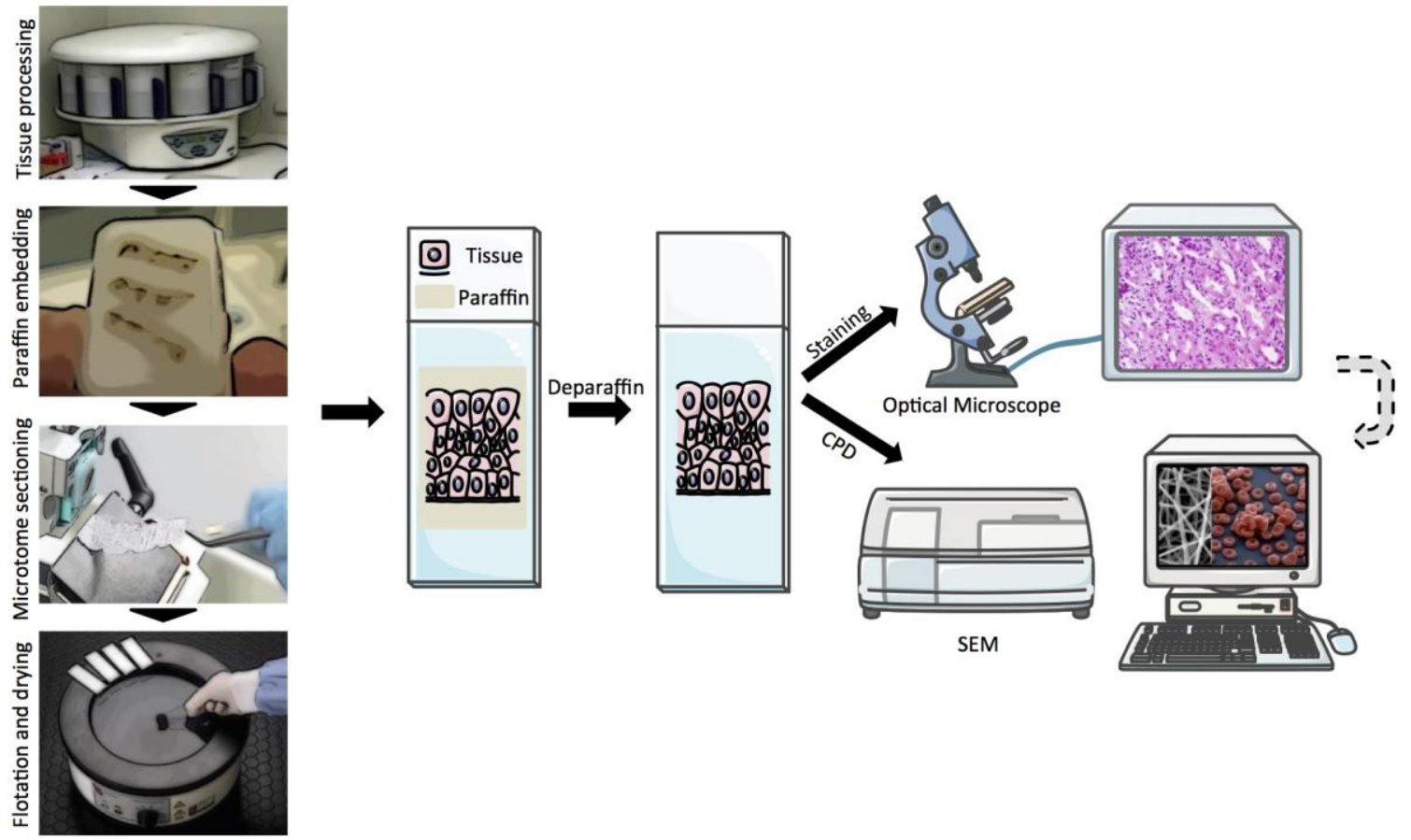

Figure 1. Schematic presentation of experimental setup showing the different steps of Histo-SEM technique used for evaluation of extracellular matrix in native and bioengineered samples. Serial sections are assessed by histological/immunohistochemical stainings, followed by light microscopy imaging, or by SEM following critical point drying (CPD). The dashed arrow indicates an alternative implementation of Histo-SEM, whereby the same section is first assessed histologically and then by SEM.

SEM is already used as a method to visualize the spatial morphology and organization of both surface and cross-sections of tissue-engineered constructs [7]. However, efforts to reach the internal space of 3D samples relies so far either on a snap freezing in liquid nitrogen or a lyophilization procedure, followed by manually breaking the construct with forceps or blades [18-20]. Such procedures result in uneven surfaces on fragile or brittle specimens. The typical deformities and fractures not only hinder acquiring high-quality images, but also practically develop electrical charges while misadjusting the contrast, hence deteriorating the information about the studied specimens [21]. This limitation was here overcome by preparing the samples with a sharp and reproducible cut of microtome following paraffin embedding. Although previous reports in botanical and agricultural area demonstrated the value of SEM 
in studying plant's sections prepared for light microscopy [22-24], Histo-SEM technique has not been reported so far in tissue repair or engineering to evaluate ECM and blood vessels organization in vitro or in vivo.

Macromass pellets formed by human articular chondrocytes used for the analyses described below were derived from a previous study [6]. We selectively assessed samples resulting in limited or efficient chondrogenesis, as assessed respectively by the absence of Safranin-O positive staining for glycosaminoglycan (Figure 2, A and D), or a dense cartilaginous matrix, positively stained for Safranin-O (in red) (Figure 2, B and E). Native cartilage tissue was used as reference (Figure 2, C and F). High-resolution SEM images of the same sections stained for Safranin-O showed no obvious alteration of the morphology and structure of the samples due to the samples' preparation procedure (Figure 2, G, H and I). The matrix of the constructs with limited chondrogenic capacity was randomly organized with several bundling and tangling (Figure 2J and Figure 3, A and B). In contrast, more chondrogenic experimental samples exhibited larger fibers with higher degree of orientation (Figure 2K and Figure 3, C and D). In native human cartilage, the ECM fibers were preferentially aligned (Figure 3, E and F), with an average diameter significantly larger (approximately four-fold) than in engineered pellets (Figure 3G). Histo-SEM allowed for a sub-micrometric comparison of ECM structural features in engineered and native cartilages. 

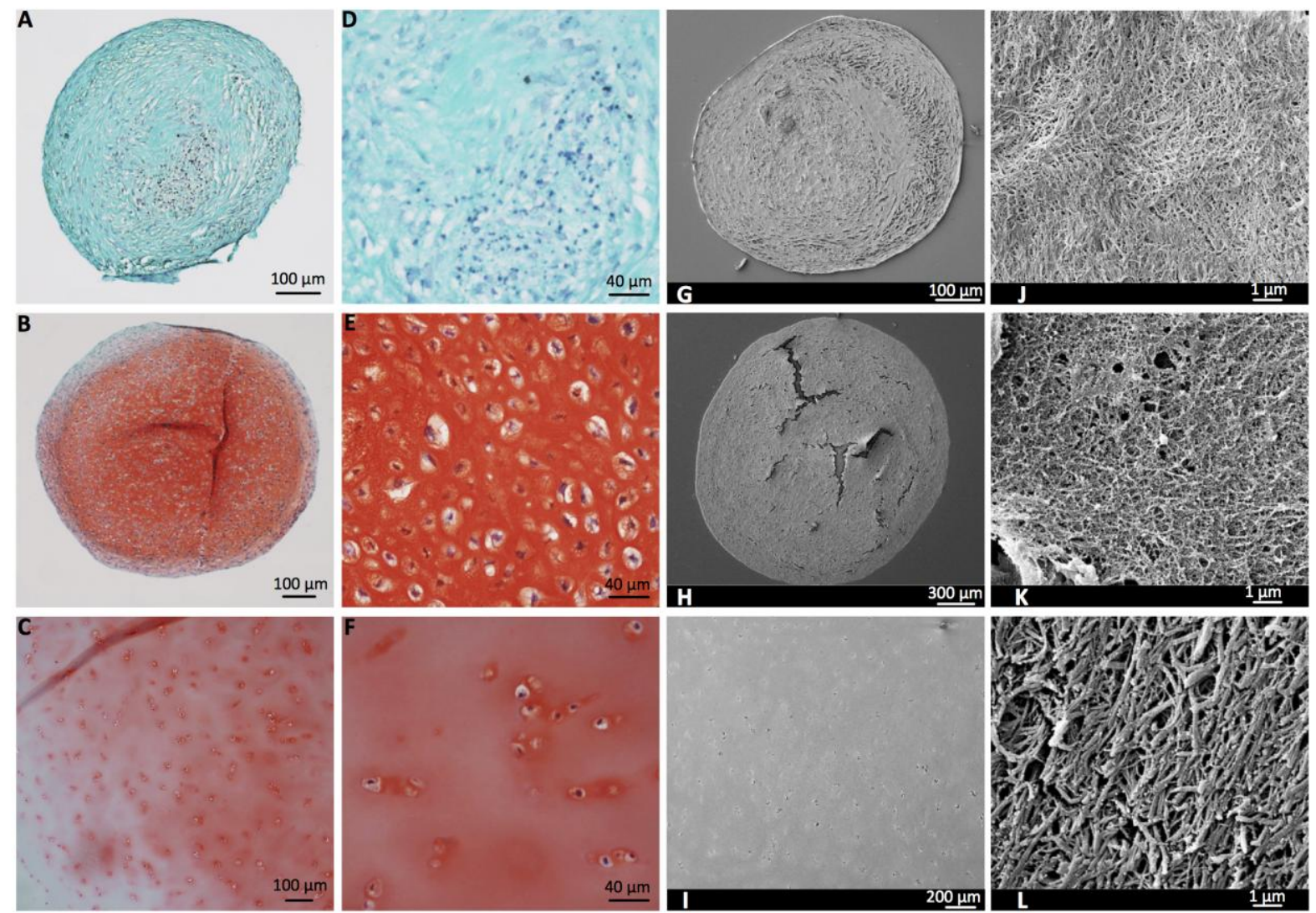

Figure 2. Glycosaminoglycan (GAG) accumulation in engineered and native cartilage tissues. Safranin-O staining of representative cross-sectional fields of pellets exhibiting limited (A, D) or efficient chondrogenesis $(B, E)$, and of human articular cartilage $(\mathrm{C}, \mathrm{F})$. Representative scanning electron micrographs of Safranin-O negative $(\mathrm{G})$ and positive $(\mathrm{H})$ pellets, and human cartilage tissue $(\mathrm{I})$, showing the structure and arrangement of the ECM nanofibrillary network (J-L). Scale bar A-C: $100 \mu \mathrm{m}, \mathrm{D}-\mathrm{F}: 40 \mu \mathrm{m}, \mathrm{G}-\mathrm{I}: 100 \mu \mathrm{m}, \mathrm{J}-\mathrm{L}: 1 \mu \mathrm{m}$.

Although Safranin-O staining did not distinguish different levels of GAG between highly chondrogenic engineered pellets and native cartilage, the sub-micrometric analysis provided by Histo-SEM revealed significant differences in the architecture of the ECM and especially of the fibrillary components of the matrix. ECM structure plays a central role in the mechanical functionality and durability of articular cartilage [25-29]. The technique could thus be further combined with mechanical tests to identify structure-function relationships distinguishing 3D engineered and native cartilage tissues. 


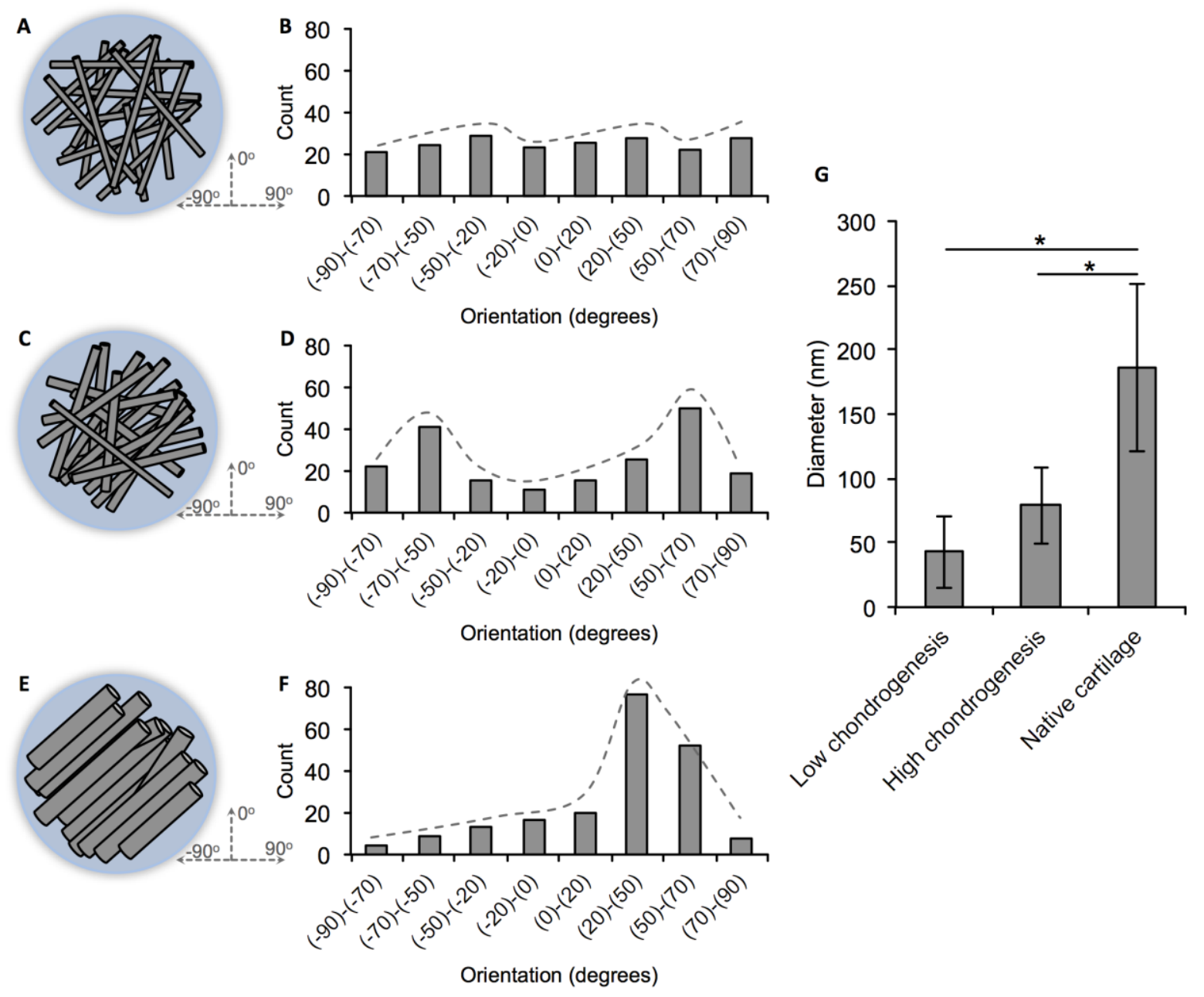

Figure 3. Structural analyses of neo formed ECM in engineered and native cartilage. Schematic representation and angular distribution of fibers in samples with low $(\mathrm{A}, \mathrm{B})$ and high chondrogenesis $(\mathrm{C}, \mathrm{D})$, and native articular cartilage (E, F). Statistical orientation analysis was performed for 200 randomly selected fibers in histological sections from $n=3$ independent samples per experimental condition. $(G)$ Average fiber diameter of samples. ${ }^{*} \mathrm{P}<0.05$.

Different microscopy techniques have been employed to detect the structural changes in engineered osteogenic constructs intended to repair bone defects [30,8,31]. In the present study, Histo-SEM was validated as an alternative method to characterize patterns of a developing bone tissue in cell-ceramic constructs. The in vivo osteogenic capacity of adiposederived cells-ceramic constructs was evaluated 8 weeks after implantation in an ectopic mouse model. Histological analysis indicated that newly-formed bone was deposited on the 
surface of the ceramic and organized in typical ossicle structures, as previously reported (Figure 4, A and B) [17]. The morphology and patterns of bone tissue and surrounding matrix were then assessed at sub-micrometric scale with the Histo-SEM technique (Figure 4B1). A non-fibrillar, highly compact, dense bone tissue was observed at the periphery of the pores, where osteocytes nested inside the matrix (white arrow, Figure 4B2). In the vicinity of the deposited bone, towards the center of the pore, arched patterns of densely packed collagen fibrils were detected, in which a well-ordered lamellar structure was organized in concentric layers parallel to the pore surface (Figure 4B3). The high-resolution [4,30,32] visualization of collagen fibers and residing cells within histologically identified regions of compact bone tissue might further help understand structural properties in more details [33], possibly distinguishing ossicles generated by different cell sources or at different stages of development.

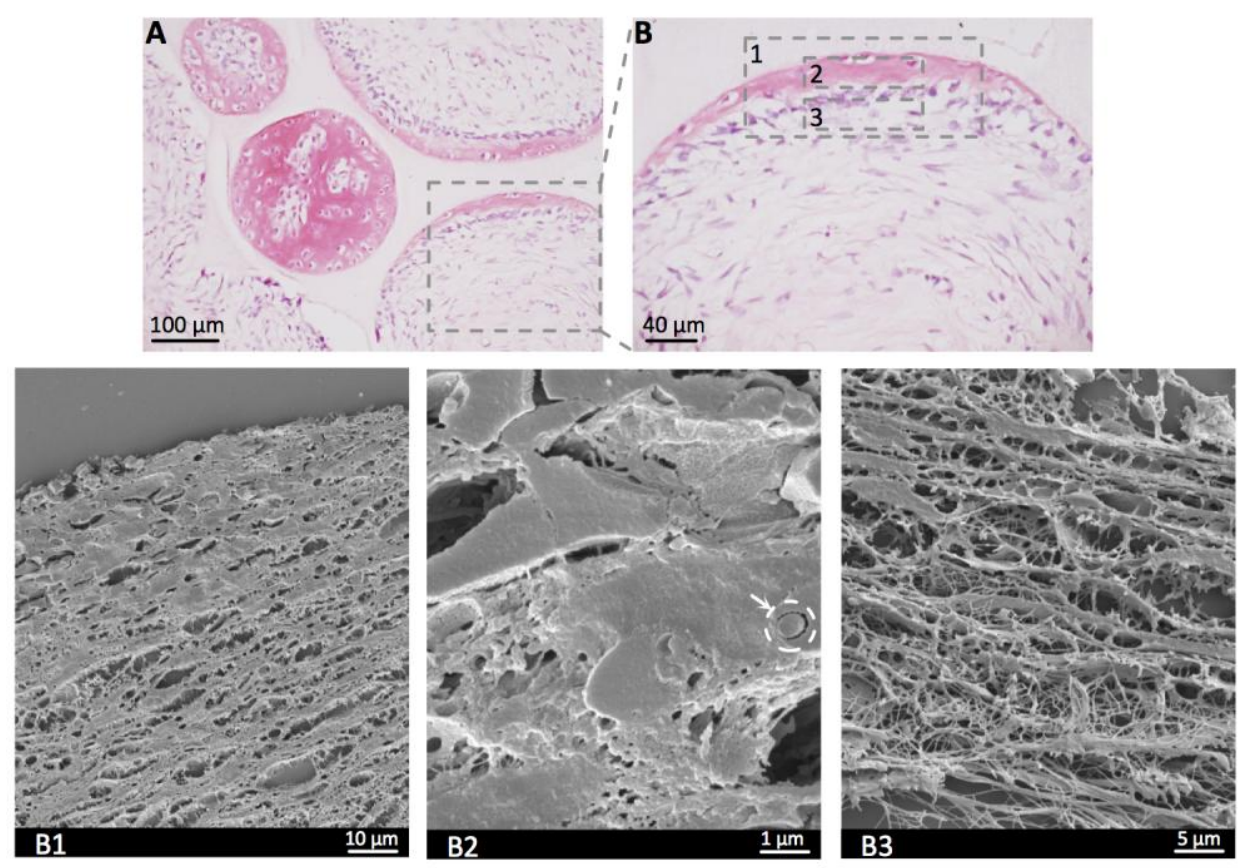

Figure 4. In vivo bone formation analyses. (A) Representative light microscopy images of H\&E stained sections of ceramic scaffolds seeded with human stromal vascular fraction cells, following 8 weeks of ectopic implantation in nude mice. (B) Higher power image better shows the two distinct regions within the construct. (B1) Representative scanning electron micrographs of the matrix deposited within the scaffold, which at higher 
magnification was displaying (B2) compact bone matrix or (B3) packed oriented collagen fibrils. The white arrow and circle indicate the presence of osteocytes within the neo-formed bone matrix. Scale bar A: $100 \mu \mathrm{m}, \mathrm{B}$ : $40 \mu \mathrm{m}, \mathrm{B} 1: 10 \mu \mathrm{m}, \mathrm{B} 2: 1 \mu \mathrm{m}, \mathrm{B} 3: 5 \mu \mathrm{m}$.

In order to evaluate the in vivo tissue ingrowth into two previously tested materials, i.e. a cross-linked type I collagen and egg-white-based sponges [5], cross-sections of the materials were stained with Masson Trichrome, observed by LM and processed for Histo-SEM analysis. Histological assessments confirmed the previously described deeper ECM accumulation inside egg white-based samples [5] (Figure 5, A and B). Histo-SEM images also indicated a similar level of tissue ingrowth into the two materials (Figure 5, C and D). Moreover, thanks to the sub-micrometric resolution, the technique allowed observing that, as compared to collagen sponge-based specimens, the ECM formed within egg-white sponges was denser (Figure 5, E and F) and with larger collagen fibers (Figure 5, G and H).
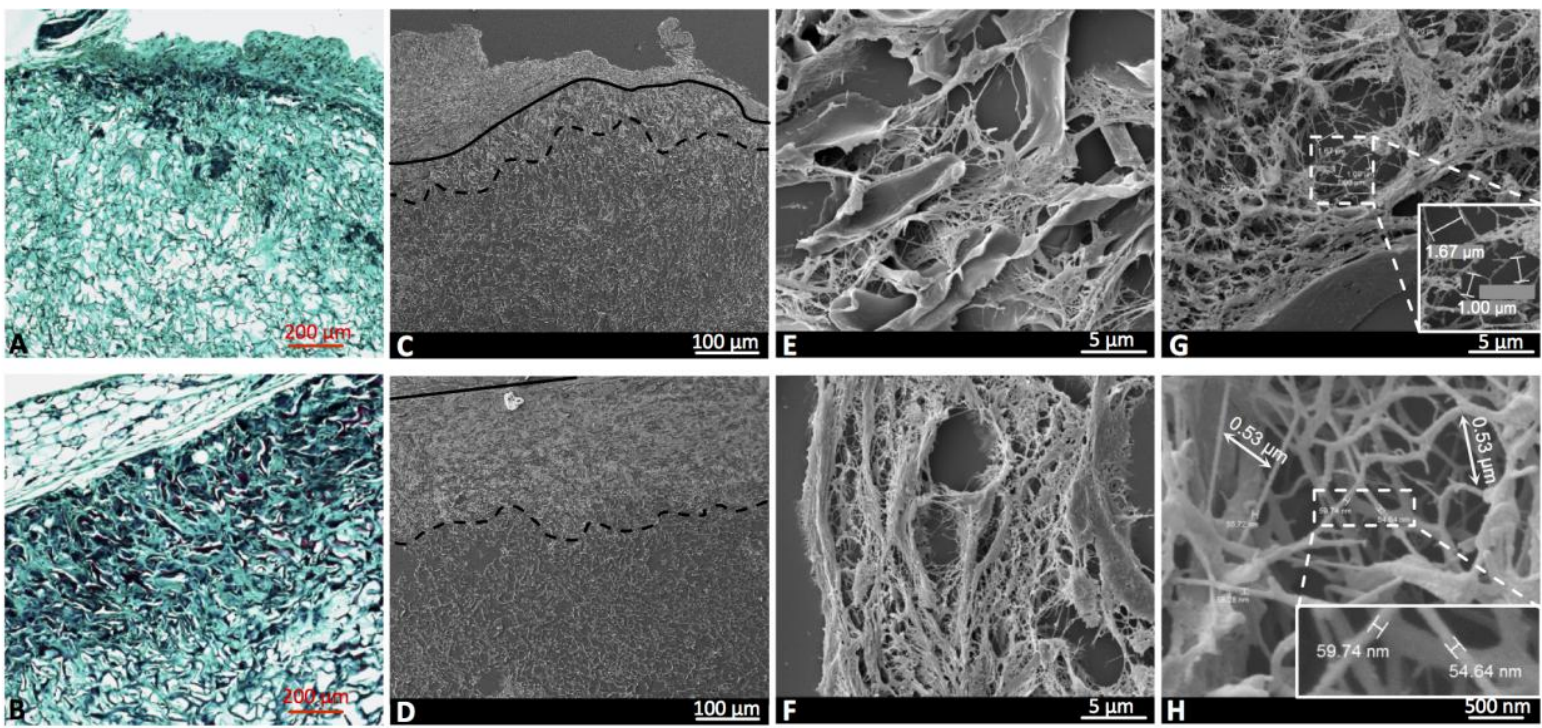

Figure 5. In vivo engraftment assessments. Micrographs of (A) collagen and (B) egg white scaffolds stained for Masson's Trichrome, following 4 weeks of subcutaneous implantation in nude mice. Scanning electron micrographs reveals the ingrowth depth and density of neo-formed matrix within the $(\mathrm{C}, \mathrm{E})$ collagen and $(\mathrm{D}, \mathrm{F})$ egg white samples. Full-line and dashed-line indicate edge of the construct and depth of tissue ingrowth, respectively. (G) Density of nanofibrous meshwork deposited within the scaffold porosities could be quantified thanks to the Histo-SEM technique. The inset shows fiber-to-fiber length measurement in a higher 
magnification. (H) Diameter of newly formed ECM fibers in the constructs could be determined using the captured scanning electron micrographs. The inset shows a high power image of fibers. Scale bar A-B: $200 \mu \mathrm{m}$, C-D: $500 \mu \mathrm{m}, \mathrm{E}-\mathrm{G}: 5 \mu \mathrm{m}, \mathrm{H}: 500 \mathrm{~nm}$.

Quantification of the distance between fibers and of the fiber diameters demonstrated the statistical significance of the qualitative observations (Figure 6, A and B). Given the depth of focus and resolution achieved by SEM [34,35], Histo-SEM demonstrated the ability to quantify the density and dimensions of the neo-formed fibrillary ECM network at the submicron level throughout the scaffolds, which cannot even be approximated by using conventional histological techniques. The extent of ECM deposition and its organization could be quantitatively compared in different engineered tissues, reflecting the extent of engraftment after in vivo implantation [36].
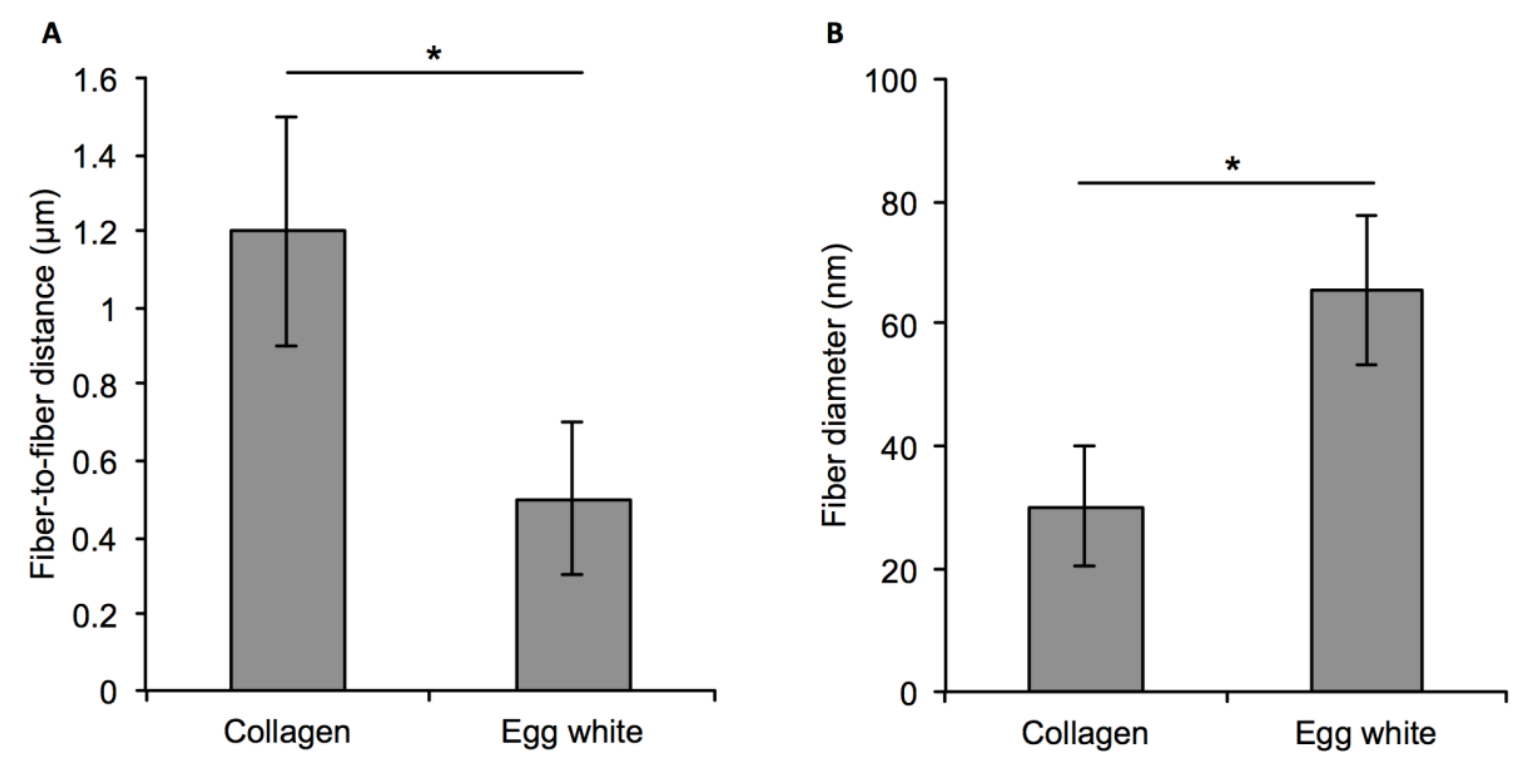

Figure 6. Quantification of in vivo neo-formed fibrillary ECM network. (A) Density and (B) average diameter of matrix fibers in collagen and egg white scaffolds. 3 independent implanted scaffolds per condition were analyzed. ${ }^{*} \mathrm{P}<0.05$.

To evaluate vascularization in implanted egg white-based sponges, sections were stained by $\mathrm{H} \& \mathrm{E}$ and immuno-histochemically for the endothelial marker CD31. LM examination 
indicated tissue ingrowth as well as blood vessels formation inside the samples (Figure 7, A and B). The Histo-SEM technique performed on the same sections further qualified the developed microvasculature. In particular, it allowed for the identification of capillaries with intraluminal red blood cells (Figure 7, C and D) within the ECM fibers (Figure 7E), and for the quantification of the vessel wall thickness, which averaged $634 \pm 102 \mathrm{~nm}$ in the assessed sample (Figure 7F). LM-based morphological assessments of vasculature are limited to metrics like diameter, ingrowth depth and perimeter length, and thus give a limited picture of the morphological features of blood vessels [37-41]. Thanks to the high magnification available in SEM micrographs, the aforementioned parameters could be measured with HistoSEM technique and provided a more robust quantitative support to compare vascular morphologies in different tissue-engineered constructs. In addition, Histo-SEM offered the possibility to study the topography of lumens and to quantify vessel walls' thicknesses. Such quantified morphological parameters might allow not only quantifying angiogenesis levels but also defining an index of vascular function, possibly related to vascular tone [1]. 

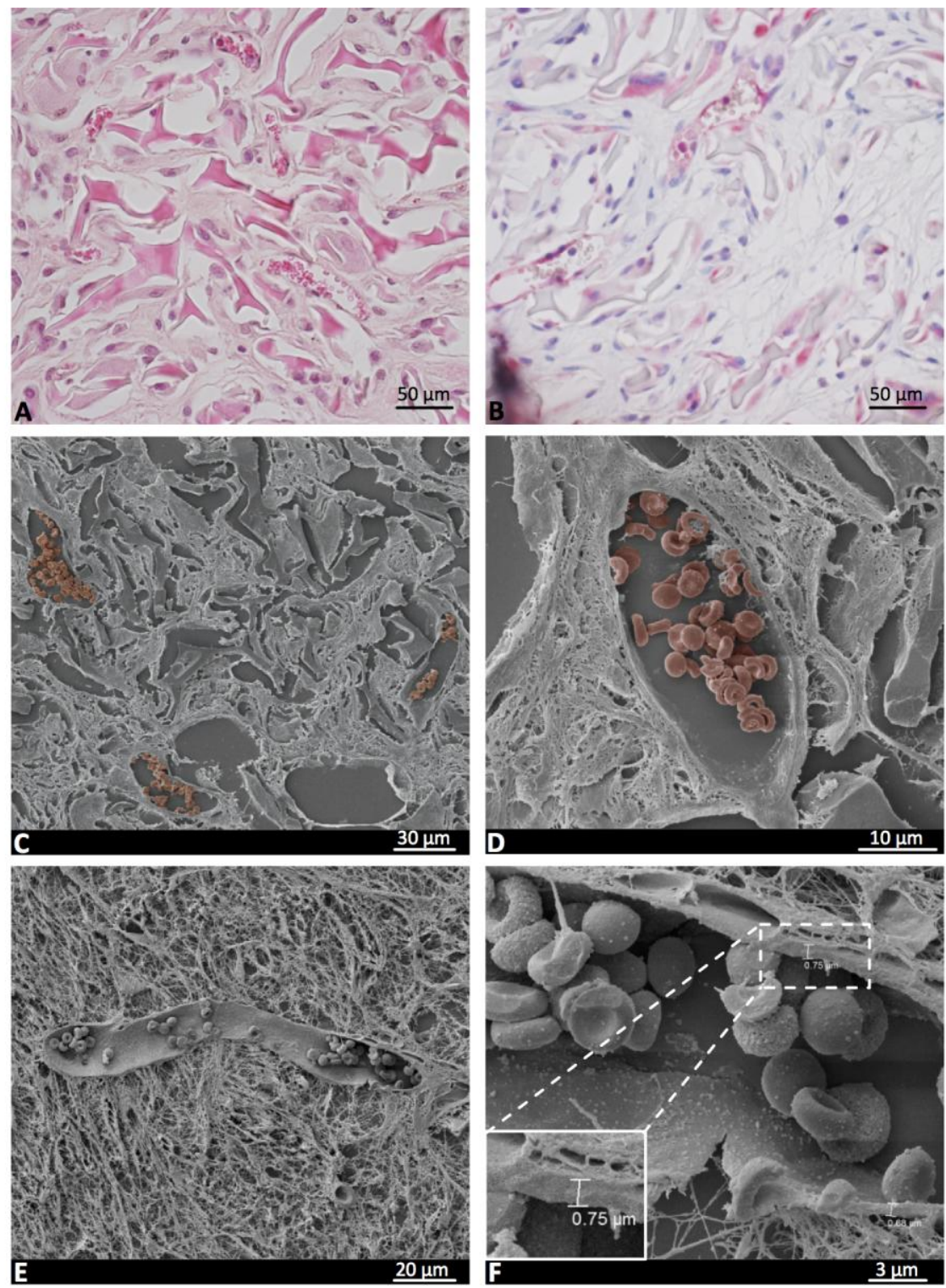

Figure 7. Angiogenesis assessments. Representative light microscopy images of (A) H\&E and (B) CD31 stained egg white scaffolds after 4 weeks of subcutaneous implantation in nude mice. (C, D) Representative scanning electron micrographs show the ingrowth of neo-vessels within the engineered construct. Erythrocytes were falsecolored in red. (E, F) Qualitative and quantitative analysis of neo-vessel's structure and morphology by HistoSEM technique. The inset shows the lumen thickness of a newly formed vessel. Scale bars in A-B: $50 \mu \mathrm{m}, \mathrm{C}: 30$ $\mu \mathrm{m}, \mathrm{D}: 10 \mu \mathrm{m}, \mathrm{E}: 20 \mu \mathrm{m}, \mathrm{F}: 3 \mu \mathrm{m}$.

Using a simple and broadly applicable procedure, called Histo-SEM, we showed how highquality micro- to nano-meter scale pictures from histological sections could help informing on the morphology of ECM or blood vessels structures, and disclosing some neglected yet 
essential parameters. The level of spatial resolution makes it appropriate also for the analysis of miniature tissue samples, such as few hundred micrometer-sized macromass pellets. It was previously proposed that SEM and LM provide complementary information about morphotopographical features of pathological tissue samples, such as those from endometriosis lesions [42]. The relevance and added value of the present method is based on the possibility to combine the assessments starting from the same or serial sections of the tissue. Moreover, it bypasses the previously reported [43] risk of deforming cartilaginous samples during SEM preparation, but also allows for a simple localization and identification of the correspondence between structures observed by the two imaging modalities. We used Histo-SEM technique to assess only paraffin-embedded tissue sections, however this method could be used for studying the entire paraffin block (i.e. without sectioning) or cryo-sectioned samples, although this was beyond the scope of the present study. Furthermore, once SEM coupled to other techniques such as energy-dispersive X-ray (EDX) spectroscopy, could provide further informative data like elemental composition of different parts of engineered tissues.

\section{Conclusion}

In summary, the results presented here validated that morpho-architectural characterization can distinguish different qualities of bioengineered constructs. The employed Histo-SEM method is able to both qualitatively and quantitatively assess the level of ECM maturation, organization of the fibrillary network or of vascular structures in native and bioengineered samples. The combination of Histo-SEM with immunohistochemical stainings may open new directions to identify the molecular nature of specific tissue structures, investigated at the submicrometric scale.

\section{Author Contributions}


S.J.-F: conception and design, collection and assembly of data, data analysis and interpretation, manuscript writing; A.S: conception and design, data analysis and interpretation, manuscript writing and editing; I.M: conception and design, manuscript writing and editing, final approval of the manuscript.

\section{Acknowledgements}

This work was supported by a Swiss excellence scholarship from the Swiss National Science Foundation to S.J.-F. (SNF grant \#2013.0022) and by a grant from the European Commission to A.S. (Marie Curie Program, Project iTERM, PITN-GA-2013-607868, http://www.itermproject.eu/home.htm). We are grateful to Ms. Evi Bieler (Zentrum fur Mikroskopie, University of Basel) for her excellent technical assistance with scanning electron microscopy. We also acknowledge Dr. Andrea Barbero for his insightful discussion on chondrogenesis analyses.

Competing interests: The authors declare no competing interests.

\section{References}

[1] J.A. Rytlewski, L.R. Geuss, C.I. Anyaeji, E.W. Lewis, L.J. Suggs, Three-dimensional image quantification as a new morphometry method for tissue engineering, Tissue Eng. Part C Methods. 18 (2012) 507-516. doi:10.1089/ten.TEC.2011.0417.

[2] R.S. Voronov, S.B. VanGordon, R.L. Shambaugh, D.V. Papavassiliou, V.I. Sikavitsas, 3D tissueengineered construct analysis via conventional high-resolution microcomputed tomography without X-ray contrast, Tissue Eng. Part C Methods. 19 (2013) 327-335. doi:10.1089/ten.TEC.2011.0612.

[3] M. Gudur, R.R. Rao, Y.-S. Hsiao, A.W. Peterson, C.X. Deng, J.P. Stegemann, Noninvasive, quantitative, spatiotemporal characterization of mineralization in three-dimensional collagen hydrogels using highresolution spectral ultrasound imaging, Tissue Eng. Part C Methods. 18 (2012) 935-946. doi:10.1089/ten.TEC.2012.0180.

[4] M.M. Villa, L. Wang, J. Huang, D.W. Rowe, M. Wei, Visualizing osteogenesis in vivo within a cellscaffold construct for bone tissue engineering using two-photon microscopy, Tissue Eng. Part C Methods. 19 (2013) 839-849. doi:10.1089/ten.TEC.2012.0490.

[5] S. Jalili-Firoozinezhad, S. Rajabi-Zeleti, P. Mohammadi, E. Gaudiello, S. Bonakdar, M. Solati-Hashjin, A. Marsano, N. Aghdami, A. Scherberich, H. Baharvand, I. Martin, Facile fabrication of egg white macroporous sponges for tissue regeneration, Adv. Healthc. Mater. 4 (2015) 2281-2290. doi:10.1002/adhm.201500482.

[6] M. Centola, B. Tonnarelli, J. Hendriks, M. van den Doel, S. Feliciano, A. Papadimitropoulos, E. Piccinini, J. Geurts, I. Martin, A. Barbero, An Improved Cartilage Digestion Method for Research and Clinical Applications, Tissue Eng. Part C Methods. 21 (2014) 394-403. doi:10.1089/ten.tec.2014.0393. 
[7] E.A. Calle, S. Vesuna, S. Dimitrievska, K. Zhou, A. Huang, L. Zhao, L.E. Niklason, M.J. Levene, The use of optical clearing and multiphoton microscopy for investigation of three-dimensional tissue-engineered constructs, Tissue Eng. Part C Methods. 20 (2014) 570-577. doi:10.1089/ten.TEC.2013.0538.

[8] S. Scaglione, P. Giannoni, P. Bianchini, M. Sandri, R. Marotta, G. Firpo, U. Valbusa, A. Tampieri, A. Diaspro, P. Bianco, R. Quarto, Order versus Disorder: in vivo bone formation within osteoconductive scaffolds, Sci. Rep. 2 (2012) 274. doi:10.1038/srep00274.

[9] S.F. Lim, R. Riehn, W.S. Ryu, N. Khanarian, C. Tung, D. Tank, R.H. Austin, In Vivo and Scanning Electron Microscopy Imaging of Upconverting Nanophosphors in Caenorhabditis elegans, Nano Lett. 6 (2006) 169-174. doi:10.1021/n10519175.

[10] R. Schilke, J.A. Lisson, O. Bauß, W. Geurtsen, Comparison of the number and diameter of dentinal tubules in human and bovine dentine by scanning electron microscopic investigation, Arch. Oral Biol. 45 (2000) 355-361. doi:10.1016/S0003-9969(00)00006-6.

[11] M. Shojaee, F. Navaee, S. Jalili-Firoozinezhad, R. Faturechi, M. Majidi, S. Bonakdar, Fabrication and characterization of ovalbumin films for wound dressing applications, Mater. Sci. Eng. C. 48 (2015) 158164. doi:10.1016/j.msec.2014.11.063.

[12] G. Camci-Unal, D. Cuttica, N. Annabi, D. Demarchi, A. Khademhosseini, Synthesis and Characterization of Hybrid Hyaluronic Acid-Gelatin Hydrogels, Biomacromolecules. 14 (2013) 1085-1092. doi:10.1021/bm3019856.

[13] P. Baei, S. Jalili-Firoozinezhad, S. Rajabi-Zeleti, M. Tafazzoli-Shadpour, H. Baharvand, N. Aghdami, Electrically conductive gold nanoparticle-chitosan thermosensitive hydrogels for cardiac tissue engineering, Mater. Sci. Eng. C. 63 (2016) 131-141. doi:10.1016/j.msec.2016.02.056.

[14] M. Simons, K. Alitalo, B.H. Annex, H.G. Augustin, C. Beam, B.C. Berk, T. Byzova, P. Carmeliet, W. Chilian, J.P. Cooke, G.E. Davis, A. Eichmann, M.L. Iruela-Arispe, E. Keshet, A.J. Sinusas, C. Ruhrberg, Y.J. Woo, S. Dimmeler, State-of-the-Art Methods for Evaluation of Angiogenesis and Tissue Vascularization A Scientific Statement From the American Heart Association, Circ. Res. 116 (2015) e99e132. doi:10.1161/RES.0000000000000054.

[15] I. Papantoniou, M. Sonnaert, L. Geris, F.P. Luyten, J. Schrooten, G. Kerckhofs, Three-Dimensional Characterization of Tissue-Engineered Constructs by Contrast-Enhanced Nanofocus Computed Tomography, Tissue Eng. Part C Methods. 20 (2013) 177-187. doi:10.1089/ten.tec.2013.0041.

[16] M. Loparic, D. Wirz, A.U. Daniels, R. Raiteri, M.R. VanLandingham, G. Guex, I. Martin, U. Aebi, M. Stolz, Micro- and Nanomechanical Analysis of Articular Cartilage by Indentation-Type Atomic Force Microscopy: Validation with a Gel-Microfiber Composite, Biophys. J. 98 (2010) 2731-2740. doi:10.1016/j.bpj.2010.02.013.

[17] A. Scherberich, R. Galli, C. Jaquiery, J. Farhadi, I. Martin, Three-dimensional perfusion culture of human adipose tissue-derived endothelial and osteoblastic progenitors generates osteogenic constructs with intrinsic vascularization capacity, Stem Cells Dayt. Ohio. 25 (2007) 1823-1829. doi:10.1634/stemcells.2007-0124.

[18] L. Wang, S. Lu, J. Lam, F.K. Kasper, A.G. Mikos, Fabrication of cell-laden macroporous biodegradable hydrogels with tunable porosities and pore sizes, Tissue Eng. Part C Methods. 21 (2015) 263-273. doi:10.1089/ten.TEC.2014.0224.

[19] L. Wang, J.P. Stegemann, Glyoxal crosslinking of cell-seeded chitosan/collagen hydrogels for bone regeneration, Acta Biomater. 7 (2011) 2410-2417. doi:10.1016/j.actbio.2011.02.029.

[20] C. Ji, N. Annabi, A. Khademhosseini, F. Dehghani, Fabrication of porous chitosan scaffolds for soft tissue engineering using dense gas CO2, Acta Biomater. 7 (2011) 1653-1664. doi:10.1016/j.actbio.2010.11.043.

[21] K.H. Kim, Z. Akase, T. Suzuki, D. Shindo, Charging Effects on SEM/SIM Contrast of Metal/Insulator System in Various Metallic Coating Conditions, Mater. Trans. JIM. 51 (2010) 1080-1083. doi:10.2320/matertrans.M2010034.

[22] Y. Mamiya, Scanning Electron Microscopy of Pine Seedling Wood Tissue Sections Inoculated with the Pinewood Nematode Bursaphelenchus xylophilus Previously Prepared for Light Microscopy, J. Nematol. 44 (2012) 255-259.

[23] J.H. Ledford, P.E. Richardson, Light and scanning electron microscopy of greenbug aphid damage in wheat using the same section, Biotech. Histochem. Off. Publ. Biol. Stain Comm. 69 (1994) 342-347.

[24] G. D.a, K. E.g, Application of scanning electron microscopy to paraffin-embedded plant tissues to study invasive processes of plant-pathogenic fungi [Coprinus psychromorbidus, wheat]., Phytopathology. (1984). http://agris.fao.org/agris-search/search.do?recordID=US19850044391 (accessed September 14, 2016).

[25] A. Changoor, M. Nelea, S. Méthot, N. Tran-Khanh, A. Chevrier, A. Restrepo, M.S. Shive, C.D. Hoemann, M.D. Buschmann, Structural characteristics of the collagen network in human normal, degraded and repair articular cartilages observed in polarized light and scanning electron microscopies, Osteoarthr. Cartil. OARS Osteoarthr. Res. Soc. 19 (2011) 1458-1468. doi:10.1016/j.joca.2011.09.007. 
[26] E.B. Hunziker, The elusive path to cartilage regeneration, Adv. Mater. Deerfield Beach Fla. 21 (2009) 3419-3424. doi:10.1002/adma.200801957.

[27] D.J. Responte, R.M. Natoli, K.A. Athanasiou, Collagens of articular cartilage: structure, function, and importance in tissue engineering, Crit. Rev. Biomed. Eng. 35 (2007) 363-411.

[28] I. Fulco, S. Miot, M.D. Haug, A. Barbero, A. Wixmerten, S. Feliciano, F. Wolf, G. Jundt, A. Marsano, J. Farhadi, M. Heberer, M. Jakob, D.J. Schaefer, I. Martin, Engineered autologous cartilage tissue for nasal reconstruction after tumour resection: an observational first-in-human trial, Lancet Lond. Engl. 384 (2014) 337-346. doi:10.1016/S0140-6736(14)60544-4.

[29] M. Bhattacharjee, J. Coburn, M. Centola, S. Murab, A. Barbero, D.L. Kaplan, I. Martin, S. Ghosh, Tissue engineering strategies to study cartilage development, degeneration and regeneration, Adv. Drug Deliv. Rev. 84 (2015) 107-122. doi:10.1016/j.addr.2014.08.010.

[30] M. Parvinzadeh Gashti, F. Alimohammadi, J. Hulliger, M. Burgener, H. Oulevey-Aboulfad, G.L. Bowlin, Microscopic methods to study the structure of scaffolds in bone tissue engineering : a brief review, in: A. Méndez-Vilas (Ed.), Curr. Microsc. Contrib. Adv. Sci. Technol., Formatex Research Center, Badajoz, Spain, 2012: pp. 625-638. http://boris.unibe.ch/53190/ (accessed August 13, 2015).

[31] A. Kaempfen, A. Todorov, S. Güven, R.D. Largo, C. Jaquiéry, A. Scherberich, I. Martin, D.J. Schaefer, Engraftment of Prevascularized, Tissue Engineered Constructs in a Novel Rabbit Segmental Bone Defect Model, Int. J. Mol. Sci. 16 (2015) 12616-12630. doi:10.3390/ijms160612616.

[32] V. Ntziachristos, Going deeper than microscopy: the optical imaging frontier in biology, Nat. Methods. 7 (2010) 603-614. doi:10.1038/nmeth.1483.

[33] A. Boyde, Scanning electron microscopy of bone, Methods Mol. Biol. Clifton NJ. 816 (2012) 365-400. doi:10.1007/978-1-61779-415-5_24.

[34] C.G. Jones, Scanning electron microscopy: preparation and imaging for SEM, Methods Mol. Biol. Clifton NJ. 915 (2012) 1-20. doi:10.1007/978-1-61779-977-8_1.

[35] A.G. Pogorelov, I.I. Selezneva, Evaluation of collagen gel microstructure by scanning electron microscopy, Bull. Exp. Biol. Med. 150 (2010) 153-156.

[36] M.P. Lutolf, J.A. Hubbell, Synthetic biomaterials as instructive extracellular microenvironments for morphogenesis in tissue engineering, Nat. Biotechnol. 23 (2005) 47-55. doi:10.1038/nbt1055.

[37] C.A. Staton, M.W.R. Reed, N.J. Brown, A critical analysis of current in vitro and in vivo angiogenesis assays, Int. J. Exp. Pathol. 90 (2009) 195-221. doi:10.1111/j.1365-2613.2008.00633.x.

[38] S.B. Fox, R.D. Leek, M.P. Weekes, R.M. Whitehouse, K.C. Gatter, A.L. Harris, Quantitation and prognostic value of breast cancer angiogenesis: comparison of microvessel density, Chalkley count, and computer image analysis, J. Pathol. 177 (1995) 275-283. doi:10.1002/path.1711770310.

[39] S.A. Ishak, J.R.P. Djuansjah, M.R.A. Kadir, I. Sukmana, Angiogenesis in tissue engineering: from concept to the vascularization of scaffold construct, IOP Conf. Ser. Mater. Sci. Eng. 58 (2014) 012015. doi:10.1088/1757-899X/58/1/012015.

[40] M.W. Laschke, Y. Harder, M. Amon, I. Martin, J. Farhadi, A. Ring, N. Torio-Padron, R. Schramm, M. Rücker, D. Junker, J.M. Häufel, C. Carvalho, M. Heberer, G. Germann, B. Vollmar, M.D. Menger, Angiogenesis in Tissue Engineering: Breathing Life into Constructed Tissue Substitutes, Tissue Eng. 12 (2006) 2093-2104. doi:10.1089/ten.2006.12.2093.

[41] S. Rajabi-Zeleti, S. Jalili-Firoozinezhad, M. Azarnia, F. Khayyatan, S. Vahdat, S. Nikeghbalian, A. Khademhosseini, H. Baharvand, N. Aghdami, The behavior of cardiac progenitor cells on macroporous pericardium-derived scaffolds, Biomaterials. 35 (2014) 970-982. doi:10.1016/j.biomaterials.2013.10.045.

[42] G. Vasquez, F. Cornillie, I.A. Brosens, Peritoneal endometriosis: scanning electron microscopy and histology of minimal pelvic endometriotic lesions, Fertil. Steril. 42 (1984) 696-703.

[43] S. Ströbel, M. Loparic, D. Wendt, A.D. Schenk, C. Candrian, R.L.P. Lindberg, F. Moldovan, A. Barbero, I. Martin, Anabolic and catabolic responses of human articular chondrocytes to varying oxygen percentages, Arthritis Res. Ther. 12 (2010) R34. doi:10.1186/ar2942. 